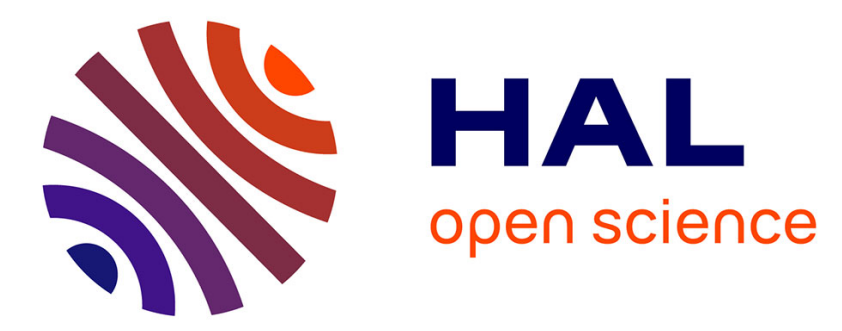

\title{
Vie temporelle et singularité selon Husserl
}

Emmanuel Housset

\section{To cite this version:}

Emmanuel Housset. Vie temporelle et singularité selon Husserl. Philosophie, 2003, Phénoménologie japonaise, 4 (79), pp.62-84. 10.3917/philo.079.0062 . hal-01871558

HAL Id: hal-01871558

https://hal-normandie-univ.archives-ouvertes.fr/hal-01871558

Submitted on 11 Sep 2018

HAL is a multi-disciplinary open access archive for the deposit and dissemination of scientific research documents, whether they are published or not. The documents may come from teaching and research institutions in France or abroad, or from public or private research centers.
L'archive ouverte pluridisciplinaire HAL, est destinée au dépôt et à la diffusion de documents scientifiques de niveau recherche, publiés ou non, émanant des établissements d'enseignement et de recherche français ou étrangers, des laboratoires publics ou privés. 


\section{Emmanuel Housset}

\section{Vie temporelle et singularité selon Husserl}

Pour madame Boudot

A quelles conditions une phénoménologie de la vie qui n'élimine pas la singularité estelle possible ? Heidegger a donné à voir une mienneté antérieure au pouvoir de dire je et cela d'abord par rapport à la transcendance du Dasein puis par rapport à l'Ereignis lui-même. Lévinas a pensé cette mienneté comma assujettissement à autrui et, plus récemment, Michel Henry l'a élucidé à partir de l'auto-affection. A chaque fois il s'agit d'envisager une mêmeté qui n'a rien à voir avec la permanence d'une substance. Mais qu'en est-il de la phénoménologie transcendantale de Husserl? Est-elle la dernière formulation d'une métaphysique de la subjectivité qui aurait toujours manqué l'individuation radicale de l'homme, ou l'ego transcendantal, comme transcendance dans l'immanence, est-il la possibilité et la nécessité de l'individuation radicale de la chair ? Si Heidegger, en élucidant le sens existential de l'ipséité dans Etre et temps, met fin à la substantialisation de l'homme et de la conscience, le fait-il par une rupture profonde avec Husserl ou n'a-t-il qu'à pousser un peu plus loin les analyses husserliennes de l'ipséité ? Dans son célèbre Nachwort, Husserl a fermement repoussé le reproche d'abstraction qui était formulé à l'encontre de son idéalisme transcendantal ${ }^{1}$. La phénoménologie transcendantale ne s'en tient pas à l'intériorité abstraite de la psychologie ou au sujet abstrait des sciences de la nature. L'ego transcendantal, comme fondement métaphysique en un sens nouveau, n'est pas un simple centre vide et intemporel comme principe d'unité d'une vie qui ne serait pas une vie parce qu'elle demeurerait finalement celle de personne. En effet, dans la mesure où l'on n'accède à la subjectivité transcendantale, comme source de toute vie, uniquement par la temporalité, qui est bien en cela l'essence même de la subjectivité, il semble difficile de dire que pour la phénoménologie transcendantale la singularité de cette vie n'a aucune importance ${ }^{2}$, tout en se demandant si une telle phénoménologie peut pleinement reconnaître une singularité qui n'est pas accessible

\footnotetext{
${ }^{1} \mathrm{Cf}$. Postface à mes idées directrices pour une phénoménologie pure : «L'on me reproche de n'atteindre en aucune façon et pour des raisons de principe la subjectivité originairement concrète », trad. franç. A.L. Kelkel, dans La phénoménologie et le fondement des sciences, Paris, PUF, col. Epiméthée, 1993, p.181.

${ }^{2}$ Voir par exemple. Michel Henry dans son livre au demeurant remarquable Incarnation, Une philosophie de la chair, Paris, Seuil, 2000, p.107: «Et voici que Husserl nous déclare que la singularité de cette vie, de la cogitatio et de son existence réelle, n'ont aucune importance ».
} 
comme objet, qui n'est pas la simple permanence d'une propriété. Non seulement chaque cogitatio se donne nécessairement comme la mienne, mais ce caractère mien est décrit dans son caractère fondamentalement paradoxal et n'est pas ici d'emblée interprété comme l'immédiateté d'une affection de soi par soi dont on peut se demander si elle ne mime pas finalement la réflexivité originaire d'un je pur. Justement parce que le temps n'est enfin plus compris comme une qualité de la chose moi, mais comme la forme, sans doute non objectivante, du rapport à soi, il n'est pas possible, sur cette question plus que sur une autre, de figer les descriptions phénoménologiques de Husserl en des formulations dogmatiques qui servent alors de repoussoir à une véritable philosophie de la vie. Il faut donc prendre au sérieux la déclaration solennelle du Nachwort en se demandant comment, avec sa rigueur propre, la phénoménologie de Husserl donne à voir un caractère mien des vécus qui n'est ni mondain, ni contingent et qui est donc tout autre chose qu'une objectivation de soi c'est-à-dire tout autre chose qu'une qualité qui s'ajoute à la chose moi. Cette question n'est donc en aucun cas une question secondaire et oubliée pour une phénoménologie eidétique, mais au contraire c'est une façon déterminante pour la recherche phénoménologique de manifester l'exigence de la donnée. Il est évident que chaque vécu se donne comme le mien et d'une façon absolument incomparable, c'est-à-dire selon un être mien qui ne présuppose pas l'intersubjectivité, qui ne soit pas simplement ce qui s'oppose à un être tien. Or, cette propriété originaire, vis-à-vis de laquelle toute altérité pourra apparaître sans être réduite plus ou moins à moi, ne peut être pensée à partir de l'alternative d'un je pur abstrait et d'un moi empirique irrationnel ou encore à partir de l'alternative d'un eidos-ego intemporel et d'une singularité temporelle contingente puisque justement le devenir soi n'est pas une simple conséquence résultant de la connaissance de soi, mais ce qui advient à même la connaissance de soi. Il s'agit donc de montrer que la conception husserlienne de l'ego transcendantal non seulement ne laisse pas de côté la question de la mienneté, mais qu'en outre sur cette question elle ne demeure pas sans arguments vis-à-vis de la compréhension de la mienneté à partir de la transcendance du Dasein, de l'exposition à l'autre ou de l'auto-affection. Un tel projet ne prend pas nécessairement la signification d'un retour à Husserl, mais cela doit permettre de situer où se trouve le lieu de fracture entre la conception transcendantale de la mienneté et la conception existentiale de la mienneté.

Husserl fait de l'élucidation du caractère absolument mien du vécu la garantie d'une rupture avec toute la métaphysique classique et la garantie d'une attention au phénomène. Il se propose, là encore, d'effectuer une réinterprétation de la Critique de la raison pure. Pour Kant, la conscience de soi fonde le caractère mien des représentations : «En effet, les représentations diverses qui sont données dans certaines intuitions ne seraient pas toutes ensemble mes représentations, si toutes ensemble elles n'appartenaient pas à une conscience de soi $^{3} »$. Husserl admet comme Kant que c'est l'unité transcendantale de la conscience qui assure qu'une représentation pourra être dite mienne, mais il va penser cette unité comme étant à la fois celle d'une réflexivité et celle d'une temporalisation et cela signifie que la mienneté transcendantale ne peut être réduite à une simple conséquence de la réflexivité. C'est en effet l'exigence d'un respect de la manifestation qui contraint à ne plus penser l'ego transcendantal à partir de la séparation de la substance et de l'accident puisqu'une telle distinction ontologique manque nécessairement le mode de donnée propre de tout vécu. Autrement dit, on peut montrer que la phénoménologie eidétique, dans sa considération de

\footnotetext{
${ }^{3}$ Critique de la raison pure: «Denn die mannigfaltigen Vorstellungen, die in einer gewissen Anschauung gegeben werden, würden nicht insgesammt meine Vortellungen sein, wenn sie nicht insgesammt zu einem Selbstbewusstsein gehöreten », Meiner, Hamburg, 1998, p.178; trad. franç. J.L. Delamarre et F.Marty, Paris, Gallimard, Pléiade, Euvres philosophiques tome 1, p.853.
} 
l'essence de la vie temporelle, ne considère pas la singularité de la cogitatio comme totalement secondaire, même s'il demeure vrai que certains textes ne prennent pas en considération cette singularité. L'ego transcendantal est tout sauf une hypostase, un substrat intemporel qui servirait de support aux vécus temporels puisque lui-même se temporalise. En cela Husserl ébranle cette conception d'un ego-subtance demeurant identique dans le temps qui est justement ce qui interdit l'accès à la singularité. Plus exactement, si l'ego transcendantal assure une permanence dans le temps, ce n'est pas qu'il soit au-delà du temps, mais parce qu'il est lui-même une durée originaire. On comprend alors que le temps est un titre qui recouvre un ensemble de problèmes «d'une difficulté exceptionnelle ${ }^{4}$ », dans la mesure où il conduit à renverser totalement la compréhension que nous avons de nous-mêmes en donnant à voir une présence à soi qui, sans être impersonnelle, n'est pas non plus mondaine et pour laquelle, jusqu'à maintenant, les noms ont fait défaut. En montrant comment l'ego qui constitue le monde se constitue en même temps lui-même, et ainsi gagne son authentique singularité, la méditation phénoménologique demeure polarisée par l'idéal d'une parfaite transparence de l'origine de toute constitution. Or, il est assez étonnant que, dans certains cas, la critique de la vision des essences, comme ce qui négligerait le soi singulier, l'existence singulière, maintienne, sous une autre forme, un tel idéal de transparence à soi qui relève pourtant directement d'une égologie transcendantale. On peut se demander, au contraire, si l'accès au caractère mien du vécu par la temporalité ne conduit pas à l'impossibilité de le réduire totalement à la seule réflexivité de l'ego. Notre être singulier estil pleinement justifiable à partir de la pure réflexivité de l'ego ou la temporalisation de soi présuppose-t-elle une autre modalité de la présence à soi ? Le paradoxe de la personne comme sujet singulier n'est-il pas dans un entrelacs d'une donation directe et d'une donation indirecte? Autrement dit, n'y a-t-il pas une singularité irréductible à l'immédiateté du voir, qui ne se laisse ni dériver, ni déduire et qui échappe en partie au travail de remémoration dans la mesure où elle n'a pas le mode d'apparaître d'un objet constitué, mais relève d'une autre forme de communication du moi avec lui-même? N'appartient-il pas à l'essence d'une vie temporelle, c'est-à-dire singulière, de ne pouvoir conduire à une telle coïncidence à soi et manquer cette non-coïncidence originaire n'est-ce pas manquer l'essence de la vie et de la singularité? Toutes ces questions montrent la nécessité et l'extrême difficulté de faire une analyse des intentionnalités très complexes qui donnent lieu à l'individuation et on ne peut que reconnaître que si effectivement Husserl s'attache surtout à élucider la forme du flux et l'individualité de l'objet temporel, on peut cependant aussi montrer qu'il ne laisse pas totalement dans l'ombre la singularité du flux quitte à prendre le risque de remettre en cause l'antériorité du je par rapport à la mienneté du flux hylétique. Il ne s'agit donc pas seulement de justifier Husserl contre l'accusation à nouveau formulée de s'en tenir à une subjectivité abstraite parce que désingularisée, mais de s'interroger aussi sur les conditions d'une effective phénoménologie de la vie singulière.

Husserl, en dévoilant le je comme fondement métaphysique, n'oublie pas de mettre ce je lui-même en question en interrogeant la façon dont il est donné à lui-même, dans une temporalisation originaire, qui se distingue radicalement de la temporalité des objets constitués, et en dégageant une identité qui n'est pas pensée avec le concept de substantialité, mais à partir de la temporalisation de soi. Dès lors, si le temps est la conscience transcendantale dans son devenir, si la vie transcendantale est le mouvement continu d'une auto-temporalisation, on ne peut élucider ce que signifie être soi sans mettre en évidence le caractère propre de la constance du sujet, sans dégager le rapport originaire du je

\footnotetext{
${ }^{4}$ Idées directrices pour une phénoménologie, (désormais : Idées 1), tome 1, trad. franç. Paul Ricoeur, Paris, Gallimard, 1950, p.274.
} 
transcendantal au temps par lequel cette constance n'est ni celle intra-temporelle de l'objet, ni la simple identité nue d'un acte intemporel. Antérieurement à ce que je me donne par le travail indéfini de réappropriation de moi, il y a un caractère propre du vécu qui ne vient pas après coup et qui, en conséquence, n'est pas la simple particularisation d'un universel, mais est la façon singulière de s'universaliser. Husserl montre à la fois qu'être soi ne résulte d'aucun étant préalable et que le je transcendantal s'éprouve comme singulier dans une temporalisation originaire. Husserl ne cesse de répéter que le je transcendantal ne meurt pas, mais il reste à comprendre le sens temporel de cette éternité puisque pour lui cette non intratemporalité de l'a priori subjectif demeure une forme de la temporalité. Dans cette perspective, la singularité peut apparaître comme un mode de donnée à soi essentiel, qui ne s'oppose plus à l'accomplissement de son essence, mais en constitue plutôt la condition de possibilité : seul «quelqu'un », qui s'apparaît absolument à lui-même dans la singularité de son pouvoir-être, peut répondre du sens du monde et non pas une simple essence. On peut contester en cela que la phénoménologie husserlienne soit toujours désingularisante, même si on peut penser que par principe une égologie ne peut laisser une vraie place à un rapport à soi antérieur au pouvoir de dire je. Cela dit, si Husserl ne décrit pas une individuation du soi livré à l'être, il décrit bien une individuation de soi à partir de la veille non réflexive d'un ego transcendantal qui n'est pas individué comme un objet est individué c'est-à-dire d'une façon contingente, mais qui est unique de par son propre mode d'être. Dès lors, même si le plus souvent Husserl a pensé la visée de soi-même comme une intentionnalité d'objet, il n'en demeure pas moins qu'en s'enfonçant dans la temporalité originaire de la subjectivité transcendantale il a envisagé le caractère mien du vécu et donc ne peut être compté comme un simple représentant d'une recherche de l'essence qui serait dépersonnalisante.

La réduction phénoménologique, en suspendant la thèse de la validité absolue du monde, libère de toute compréhension mondaine du sujet et dénonce le projet d'une connaissance exhaustive de son soi mondain, de ce qui est à moi, comme dépourvu de sens. Non seulement la connaissance de mon existence comme chose du monde ne me donnerait pas accès à mon identité la plus propre, mais encore la seule recherche psychologique, loin de révéler une identité, fait éclater celle-ci en une infinité de parcelles qu'il est impossible de rassembler en une unité. Seule une question en retour sur la subjectivité absolue peut sauver de l'anonymat dans lequel enferme toute considération mondaine de soi en égarant dans une recherche indéfinie de ce qui est à soi et non soi. Ainsi, la mise entre parenthèses de toute référence à un moi transcendant libère d'un anonymat radical lié à l'acte de prendre une identité secondaire et dérivée pour une identité absolue. Il ne s'agit pas de perdre sa singularité pour s'en tenir à une description des structures de la conscience pure, pas plus que la réduction n'est une perte du monde objectif, mais du souci de se libérer de toute compréhension de soi comme étant la permanence d'une substance : la singularité du soi ne se donne pas comme la singularité de l'objet.

Certes, les analyses husserliennes ne partent pas directement d'une étude du statut ontologique de l'ego, mais interrogent d'abord ce que signifie pour un objet être pour la conscience. Cela dit, c'est bien cette perspective d'abord noématique qui conduit à formuler l'exigence absolue de ne pas expliquer le monde à partir d'une chose du monde. En effet, en allant du monde à un ego origine du monde, Husserl peut montrer en quoi le moi humain n'est pas une donnée absolue, mais une donnée problématique et obscure et qu'il convient d'être attentif à son mode d'apparaître. En effet, le projet premier de Husserl demeure celui d'une critique radicale de la connaissance qui veut accéder à la pureté de l'apparaître et ne peut donc maintenir la validité d'un moi transcendant. Cependant, si le retour à la sphère de 
l'immanence, c'est-à-dire à la donation absolue, conduit à penser une conscience sans âme, il ne s'agit pas pour autant d'une conscience anonyme. La radicalité de la réduction conduit certes à s'arracher à soi comme moi empirique pour s'apparaître comme origine du sens, mais elle ne réduit toute mienneté comprise comme complexe de qualités réales que pour donner à voir une identité purement immanente qui doit avoir son propre mode de donnée et qui relève d'une connaissance non objective. Il y a une qualité du flux du vécu qui ne peut pas, par principe, être objectivée comme l'identité d'une chose, comme l'identité de ce moi humain. Ce caractère mien du flux senti, éprouvé, ne peut pas être confondu avec la qualité objective des choses parce qu'il est justement originaire, réel, et non obtenu au terme d'un long travail de reconstruction. Ainsi, en suspendant l'objectivation empirique spontanée de soi, la réduction phénoménologique bouleverse la façon habituelle de se comprendre, brise la familiarité avec soi, en interdisant de reconduire l'appréhension de soi à l'apparaître du monde.

Or, la difficulté d'une telle rupture avec la conception traditionnelle du caractère mien de la représentation se marque par le fait que celui qui a initié le projet de penser une conscience sans âme retombe dans une détermination mondaine du mien : «Nous avons besoin ici de la réduction, afin que l'évidence de l'existence de la cogitatio ne se trouve pas confondue avec l'évidence que ma cogitatio, la mienne, existe, avec celle du sum cogitans, etc. ${ }^{5} \gg$ Descartes, en maintenant une parcelle de monde maintient finalement la totalité du monde qui occulte l'être de la conscience. En comprenant l'ego comme psyché, Descartes a manqué de radicalité et n'a pas satisfait à l'exigence de la donnée absolue : il a confondu la source de toute donation avec ce qui apparaît en premier, avec le vécu psychologique et le moi auquel il appartient. Or cet échec interdit l'entrée à ce que Husserl nomme le pays des phénomènes purs. Descartes aurait ainsi maintenu le préjugé fondamental qui conduit à manquer la fonction transcendantale de l'ego. Certes, on a pu objecter que Husserl n'a pas véritablement saisi le sens du projet cartésien en ne voyant dans les Méditations métaphysiques que l'idéal d'une science absolument fondée dans l'ego pur. Descartes ne visait sans doute pas à dégager la fonction transcendantale de la subjectivité et il ne peut donc avoir échoué dans un projet qui n'était pas le sien. Cependant, même si Husserl, pas plus que Kant, n'a pensé la finitude du «je pense» du fait même de la clôture sur soi de la subjectivité transcendantale qui ne l'expose radicalement à aucune contingence, on ne peut pas non plus réduire l'ego transcendantal husserlien au simple statut d'une condition de possibilité de la science même si c'est l'une de ses premières préoccupations. Husserl est bien conscient qu'une telle tentation est issue de la réduction du moi empirique. En effet, cette réduction est bien la plus difficile parce qu'elle semble me faire perdre toute réalité, toute identité personnelle, à tel point que l'expression «moi-même» peut sembler désormais vide de tout sens. Il ne m'est même plus possible de parler de «ma» cogitatio parce quand je parle de «ma» perception de cette table le pronom possessif reconduit en fait au moi mondain, au moi ici et maintenant dont l'individuation est définie comme une limitation. Ainsi, c'est la réduction qui libère le je transcendantal de toute objectivation et le rend à son statut ontologique d'un pouvoir-être infini, d'une tâche d'être infinie.

La réduction n'est donc pas un processus d'abstraction qui isolerait l'âme du corps, parce que ce processus d'abstraction maintient dans la dépendance vis-à-vis du monde. Or, en montrant que la saisie de la cogitatio comme la mienne a spontanément lieu sur l'horizon du monde, la phénoménologie peut légitimement former le projet de rendre visible une identité qui n'a pas été vraiment interrogée dans les philosophies antérieures. Désormais l'identité ne va plus de soi et le résultat, provisoire, de la réduction est de conduire à une conscience non

${ }^{5}$ L’idée de la phénoménologie, trad. franç. Alexandre Lowit, Paris, PUF, 1970, p.68. 
personnelle, non intra-temporelle et non substantielle puisqu'un vécu ne peut plus être dit mien en se référant à un moi mondain singulier et isolé : pour que la perception soit donnée comme un phénomène pur, il faut «laisser le rapport au moi de côté ${ }^{6} »$. Or cette conscience, loin de faire perdre le monde, est ce qui le donne à voir à titre de phénomène et ce qui donne accès aux choses dans leur altérité, c'est-à-dire dans leur phénoménalité propre. Il s'agit donc maintenant de montrer comment l'ego transcendantal se connaît lui-même, s'apparaît à luimême, sans pour autant s'objectiver. Il reviendra justement à l'analyse de la temporalité de montrer que le principe de toute représentation est lui-même «re-présentable» d'une façon qui lui est propre. Il sera alors possible de remettre en cause l'idée que la «mésinterprétation du cogito cartésien par Husserl» conduise nécessairement au «dénigrement de la vie singulière ${ }^{7} »$. La description de la temporalisation originaire de soi ne dévoile-t-elle pas aussi chez Husserl une vie transcendantale qui est une vie singulière avant toute remémoration de soi ? Husserl ne se heurte-t-il pas également à la singularité d'un pouvoir-être que le sujet éprouve constamment dans un sentir originaire, mais qui ne lui apparaît qu'indirectement qu'à partir de sa tâche infinie de temporalisation, de possibilisation $?^{8}$ Il est certain que les leçons de 1907, publiées sous le titre L'idée de la phénoménologie, n'expliquent pas comment «ma » cogitatio est bien la mienne en dehors de toute référence au monde, mais elles ne sont pas le dernier mot de Husserl et elles ouvrent à la mise en évidence d'une identité comme mouvement vivant de temporalisation de soi qui a lieu d'abord dans la pure passivité. Sur ce point précisément, il y a bien chez Husserl une remise en cause de la distinction simple du fait et de l'essence. N'appartient-il pas à l'apparaître propre du moi transcendantal qu'il ne soit pas donné à lui-même comme une simple essence, mais avant tout comme la singularité d'une tâche de temporalisation, même si Husserl n'a pas vraiment élucidé d'où une telle tâche pourrait m'être donnée en propre.

La mise hors circuit du moi empirique est à comprendre comme un geste de méthode qui donne à voir un caractère mien du vécu plus originaire, non relatif au monde, et sans lequel la singularité mondaine est inintelligible. Cela suppose de s'arracher au temps objectif pour décrire la temporalité immanente des vécus. Mais, plus radicalement encore, cette méthode est une ouverture sur la vie des choses, qui sont par essence des unités de durée, et sur la vie du sujet dans l'infinité de sa possibilisation. En effet, par la mise en évidence des actes qui constituent l'identité temporelle de l'objet (il est appréhendé comme le même dans ses changements), Husserl dégage également le principe d'unité de la vie intentionnelle, qui rend chaque vécu mien. De ce fait, l'intentionnalité de la conscience du temps n'est pas une forme particulière de l'intentionnalité, mais sa forme même : toute conscience, fut-elle un sentir, est par principe temporelle et pas seulement la conscience d'un objet.

Toute la difficulté de cette description des Leçons pour une phénoménologie de la conscience intime du temps est de s'en tenir à la donnée absolue de la durée apparaissante de façon à penser la réalité du temps en ne réduisant plus le maintenant à l'instant mathématique, qui n'est qu'une construction de l'entendement. Partir de la donnée du temps conduit à montrer, dans ces leçons de 1905, qu'il n'y a pas de perception d'une durée sans qu'il y ait également une durée de la perception. Il n'y a donc pas de subjectivité intemporelle qui

\footnotetext{
${ }^{6}$ L'idée de la phénoménologie, p.69.

${ }^{7}$ Cf. Michel Henry, Incarnation, une philosophie de la chair, §12, Paris, Seuil, 2000, p.103.

${ }^{8}$ Pour Michel Henry, l'eidétique en manquant la singularité de la présence à soi manque la vie : «A la vie appartiennent des traits décisifs que nous avons reconnu, le fait que toute vie s'éprouvant soi-même dans une épreuve de soi effective et comme telle singulière porte nécessairement en elle un soi singulier, de sorte qu'elle est une vie singulière, celle de telle moi particulier », Incarnation, une philosophie de la chair, p.107.
} 
surplomberait les objets temporels : la temporalité des objets immanents reconduit à la temporalité plus originaire des actes intentionnels (perception, souvenir), et celle-ci reconduit à la temporalité de la conscience absolue qui est en deçà des vécus intentionnels. Ce résumé des trois grandes étapes des Leçons montre que l'analyse phénoménologique cherche à remonter du temps apparaissant à la temporalité originairement constituante, qui est l'origine de toute vie et est elle-même une vie.

Pour expliquer l'unité de la durée écoulée d'une mélodie, Husserl remonte à la continuité des phénomènes d'écoulement qui ont leur propre mode d'apparaître. Il montre ainsi, avec le diagramme du temps, que la durée des modes d'écoulement de la durée d'une mélodie inclut la continuité des modes d'écoulement de chaque point de cette durée. Or, si chaque phase d'une mélodie est en elle-même une continuité de passés, cela signifie que dans le flux de la conscience intime du temps il n'y a jamais rien de figé. La continuité même du temps résulte donc pour Husserl d'une double continuité : d'un côté il y a le surgissement continu des impressions originaires et de l'autre côté il y a la continuité de la rétention comme conscience originaire du tout juste passé. La rétention est véritablement l'unité intentionnelle du présent et du passé, même si elle ne procède pas de l'activité de l'ego, puisqu'elle a lieu dans le cadre de la pure passivité, comme Husserl le montrera après 1905. C'est en devenant une conscience rétentionnelle que la conscience impressionnelle devient intentionnelle et que la subjectivité porte en elle «l'héritage ${ }^{9} »$ d'un passé qui porte en lui la vie d'une modification continue. Loin d'être un simple phénomène régressif, la rétention est ce qui assure le lien de tous les vécus au présent vivant de la subjectivité ; lien sans lequel les vécus ne pourraient pas être dits miens. Husserl parvient donc à donner à voir une fondamentale unité du flux des vécus qui est antérieure à tout acte intentionnel de la remémoration. Cet être mien de la temporalisation originaire est un pur rapport à soi, non réflexif, qui est totalement indépendant vis-à-vis de toute position de l'existence d'un ego-chose. Ainsi, en étant aussi continue que l'impression, la rétention rend possible un lien intuitif avec le passé sans lequel il n'y aurait ni objet mien, ni vécu mien. Plus encore, c'est ce lien intuitif qu'est la rétention qui rend possible l'acte du ressouvenir par lequel un moment passé est donné comme ayant été présent.

Dès lors, pour Husserl, dire que le présent est le temps primordial ne consiste vraiment plus à dire que seul le présent est et que le passé et le futur sont frappés par un manque d'être. Bien au contraire, la rétention montre justement en quoi le passé «est». En montrant ainsi que le passé est tout autre chose qu'un présent qui n'est plus, puisqu'il continue à se donner, Husserl met fin à une métaphysique qui fige l'être du temps dans le présent et du même coup méconnaît le caractère temporel de la présence à soi. Avant tout acte de remémoration, qui objective chaque vécu comme le mien, à partir du telos d'une transparence absolue et d'une parfaite maîtrise de soi et du temps, chaque vécu est déjà mien de par la différence rétentionnelle comme présence originaire à soi : «Le remémoré apparaît comme ayant été présent, et ce de façon immédiatement intuitive ; et il apparaît ainsi grâce au fait qu'apparaît intuitivement un présent qui est à distance du présent actue ${ }^{10} \gg$. Dans cette communication immédiate avec les données hylétiques en dehors de toute référence à un objet perçu, qui est de l'ordre du pathique, la subjectivité se sait riche d'un héritage où elle s'éprouve comme la même avant même de dire je et pour pouvoir le dire. Le flux phansiologique est individué, mais pas au sens habituel et c'est pourquoi il ne se laisse pas objectiver comme un

\footnotetext{
${ }^{9}$ Leçons pour une phénoménologie de la conscience intime du temps, trad. franç. Henri Dussort, Paris, PUF, 1964,(désormais Leçons) p.44 : «Chaque rétention est en elle-même modification continue, qui porte en elle pour ainsi dire, dans la forme d'une suite de dégradés, l'héritage du passé ».

${ }^{10}$ Leçons, p.78.
} 
processus ${ }^{11}$. Il s'agit pour Husserl de montrer comment le pouvoir de dire je et le pouvoir de se rassembler soi-même en une histoire reconduisent à une vie où je suis déjà devenu comme le même, où chaque maintenant s'éprouve comme marqué par une identité absolue : «Le point sonore, dans son individualité absolue, est maintenu dans sa matière et dans sa situation temporelle, cette dernière seule constitue l'individualité ${ }^{12} »$. Husserl peut alors penser une individuation en dehors de l'objectivation et une identité du flux qui n'est pas du même ordre, par principe, que l'individualité de l'objet temporel : «Et de même aussi pour ce flux entier que je peux identifier de manière propre comme ce même flux. Mais cette identité n'est pas l'unité d'un être constant et ne pourra jamais le devenir ${ }^{13}$ ». Même si Husserl, dans les Leçons, se préoccupe plus de la forme du flux que du caractère mien du flux du présent, on ne peut pas dire qu'il n'envisage pas ce «comment» où il y va de nous-mêmes tout entiers. Ainsi, être le même ne signifie plus, comme pour Kant, la coïncidence d'un acte pur et intemporel à travers le flux des représentations, mais reconduit à l'unité d'une individuation continue à travers la constitution de l'expérience: le sujet temporalisant est lui-même temporalisé, sans qu'il y ait pour autant cercle, puisque cette première constance de soi dans la rétention est une auto-affection de la conscience qui produit une individuation peut-être plus radicale que celle que je peux me donner par réflexion.

La description des vécus constituant le temps se concilie avec une philosophie de la réflexion polarisée par l'idée d'une parfaite compréhension de soi, par une pleine transparence à soi. En effet, par la rétention le flux du vécu est déjà le mien, mais cette unification des vécus par le lien intuitif au présent fonde aussi la réflexion comme notre permanente possibilité : toute cogitatio antérieure peut devenir l'objet d'une perception immanente. Toute réflexion en un sens présuppose la conscience intime du temps puisque ce qui se donne à la réflexion exige que le sujet se tourne vers ce qui était déjà là avant que son regard ne se tourne vers lui : «Ce qui dans la réflexion est saisi de façon perceptive se caractérise par principe comme quelque chose qui non seulement est là et dure au sein du regard de la perception, mais était déjà là avant que ce regard ne se tourne dans sa direction ${ }^{14}{ }$. Il y a là, cette fois, une étrange circularité puisque la réflexivité dérive de la conscience intime du temps que, d'un autre côté, elle rend possible. En effet, la réflexion suppose un héritage de vécus passés qui ont une aptitude de principe à être perçus, à être réflexivement saisis. En cela, par la rétention, il y a un premier caractère mien du passé qui rend possible sa réappropriation dans le souvenir parce que le sentir, même s'il n'est pas un acte volontaire, est déjà mon acte en tant qu'il reconduit à l'ego comme pôle identique : «Moi qui perçois, je suis identiquement le même qui ensuite juge ou sens, désire, veut, et ne serait-ce qu'à cause de cette identité, je peux dire que tous sont mes actes ${ }^{15} \gg$. C'est donc bien elle qui libère d'une conscience intemporelle et anonyme et reconduit à la temporalisation vivante de soi. Dès lors, si la rétention «est une modification intentionnelle (qui a lieu) dans le cadre de la pure passivité $^{16} »$, cette continuité de la rétention est déjà en quelque sorte individuante et rend possible la singularité issue de l'action même du je. En un tout autre sens que pour Heidegger, on peut dire qu'ici la mêmeté de la temporalisation passive précède le je de la réflexion. Même s'il est incontestable que la thèse la plus constante et bien connue de Husserl est que l'égoïté rend possible l'ipséité, il n'en demeure pas moins qu'un vécu ne peut faire l'objet

\footnotetext{
${ }^{11}$ Voir Die Bernauer Manuskripte über das Zeitbewusstsein (1917/1918), Husserliana XXXIII, Kluwer Academic Publishers, Dordrecht/Boston/London, 2001, p.207.

${ }^{12}$ Leçons, p.87.

${ }^{13}$ Leçons, p. 151.

${ }^{14}$ Idées I, trad. franç. Paul Ricoeur, Paris, Gallimard, 1950, § 45, p.146.

${ }^{15}$ De la synthèse passive, trad. franç. B. Begout et J. Kessler, Grenoble, Millon, 1998, p.37.

${ }^{16}$ Expérience et jugement, § 23b, trad. franç. D. Souche, Paris, PUF, 1970, p.129.
} 
d'une reprise réflexive et devenir véritablement mien que si la rétention maintient le lien intuitif avec ce vécu inséparable, par la rétention, de mon flux de conscience. Plus encore, «c'est justement une loi originaire qui veut que chaque écoulement rétentionnel - dans la pure passivité, sans participation du moi actif - motive aussitôt et constamment des intentions d'attentes et par là même les produit ${ }^{17}$ ». Ce «style de $1^{\prime \prime}$ à venir"18 » manifeste aussi cette singularité du flux en ce qu'il dessine le comment d'un advenir. En conséquence, la conscience ne peut se saisir elle-même qu'en tant qu'elle est déjà devenue : le se sentir de la conscience rétentionnelle rend en quelque sorte possible le fait de s'advenir dans la réflexion.

Cela dit, Husserl ne renonce jamais vraiment au caractère égologique de la mienneté : si les vécus du temps ne sont plus les qualités objectives d'un moi empirique qui serait le mien, ils demeurent les miens en un nouveau sens, à savoir en tant qu'un je pur, qui a sa propre temporalité, est inséparable d'eux. Ce je pur, qui n'est donc plus à confondre avec le moi phénoménologique constitué dans l'immanence, ou avec une identité nue et intemporelle, qui n'est qu'une forme abstraite de ce moi phénoménologique, est inséparable de chaque pensée sans se confondre avec aucune. Chaque flux de conscience possède en cela une unité hors de toute comparaison, puisque ce je pur, qui fonde le caractère mien du vécu, est un pouvoir a priori de réactiver ce vécu : «Tous (les vécus de l'arrière-plan) en tant qu'ils appartiennent à un unique flux du vécu qui est le mien, doivent pouvoir être convertis en cogitationes actuelles ${ }^{19} »$. Ici, le je pur, comme transcendance dans l'immanence, laisse apparaître un caractère mien du vécu comme saisie toujours possible par un ego et non comme la mêmeté d'une temporalisation passive. Certes, il n'est pas à comprendre comme une simple persistance dans le temps, mais comme une veille d'un sujet qui peut toujours revenir à cette temporalisation première qui ne cesse jamais, même dans le sommeil. La perspective est ici encore plus noématique que noétique, et Husserl n'interroge pas vraiment dans les Idées I le caractère mien des actes qui constituent la subjectivité. Cependant, en décrivant le je pur comme la possibilité inconditionnée de prendre conscience de soi, il dévoile bien une distinction du mien et du non mien qui n'est pas mondaine et qui ne présuppose pas non plus l'intersubjectivité. Ici est mien ce qui dépend d'abord de ma maîtrise possible et non il est vrai ce qui provient d'une vie passive où la subjectivité se reçoit de ce qui l'affecte dans l'impression originaire.

Pour Husserl, le je pur n'étant pas une qualité objective du cogito, mais sa vie même, il est la source de toute temporalisation de soi, même s'il ne dure pas comme les vécus durent : «seuls des actes de l'expérience réflexive nous révèlent quelque chose du flux du vécu et de sa nécessaire référence au je pur $^{20} »$. Autrement dit, c'est toujours déjà à partir de la mienneté active qu'on accède à la mienneté passive. Ainsi, l'individuation comme temporalisation est tout autre chose qu'une limitation et elle prend le sens d'un pouvoir infini de saisie réflexive qui s'enracine dans une épreuve originaire de soi comme condition a priori de toute expérience. L'éveil à soi est alors la récupération de toute la vie passive de façon à se l'approprier et de ce fait je suis plus moi-même dans l'activité que dans la passivité, dans la veille que dans le sommeil ${ }^{21}$. L'égoïté fonde ici une ipséité comme identité d'un acte, qui ne peut être saisi indépendamment de ce qu'il effectue, c'est-à-dire de la temporalité immanente qu'il constitue. En cela le je pur ne peut pas être compris comme un simple substrat

\footnotetext{
${ }^{17}$ De la synthèse passive, p.74.

${ }^{18}$ Ibid..

${ }^{19}$ Idées I, § 57, p.190.

${ }^{20}$ Idées I, § 78, p.256.

${ }^{21}$ Sur la question du sommeil chez Husserl, voir Pierre Carrique, Rêve, Vérité. Essai sur la philosophie du sommeil et de la veille, Paris, Gallimard, 2002.
} 
intemporel puisqu'il n'est pas ce qui demeure inchangé dans le temps. Ce je pur est l'acte par rapport auquel demeurer le même et ne pas demeurer le même peuvent prendre sens. En effet, n'étant ni séparable des vécus, ni enfermé dans les vécus, le je pur est bien une transcendance dans l'immanence de la temporalité. Certes, cela ne répond pas encore pleinement à la question du mode d'apparaître du je pur, mais Husserl peut de cette façon montrer que la temporalité est «une forme nécessaire qui lie des vécus à des vécus ${ }^{22}$ ». Un vécu n'est donc mien que s'il est temporel : " chaque vécu en tant qu'être temporel est le vécu de son je $\operatorname{pur}^{23} »$. La réflexivité de l'ego, comme principe d'une centration du rapport à l'être, fonde donc bien l'unicité de la temporalité immanente, mais en tant qu'elle est elle-même une temporalisation originaire. On peut donc bien dire que la temporalisation active et la temporalisation passive sont les deux formes de la mienneté entre lesquelles cela ne va pas sans tensions : la temporalisation passive en un sens dispose de moi, son orientation et son style me précèdent, mais c'est la temporalisation active qui demeure ultimement individuante. De ce point de vue, toute la méditation sur la vie passive originaire, par exemple celle du sommeil, n'a d'autre but que de nous permettre de ne pas faire de notre vie un sommeil, que de nous permettre de vivre notre vie au lieu de la subir.

La très grande force des analyses husserliennes du temps est précisément qu'elles ne se contentent pas de reconduire «mon » flux de conscience à une réflexivité anonyme qui en étant celle de tout homme n'est celle de personne. Tout au contraire, la réponse à l'objection du solipsisme commande d'accéder à l'expérience du «propre », selon l'expression de la cinquième des Méditations cartésiennes. En effet, cette épreuve de ce qui m'est originairement propre est une condition a priori de toute expérience d'une identité et d'une altérité. Un je pur compris comme un simple acte intemporel ne peut que transformer l'expérience des choses en l'expérience d'un objet «abstrait» qui a perdu toute son altérité. Un tel je est notamment celui de la physique : «on ne remarque donc pas à quel point il est absurde de porter à l'absolu la nature physique qui n'est que le corrélat intentionnel de la pensée déterminant logiquement son objet ${ }^{24} \gg$. La nature est ainsi ce qui est produit par une conscience théorique désingularisée, abstraitement universelle, qui détermine à l'avance les objets dont elle peut faire l'expérience. Ici, on perd en même temps et la singularité et la vie du sujet et du monde. En effet, un je pur formel et vide «réduit » toute expérience du monde à une expérience de soi, exactement comme le fait le simple sujet théorique des sciences de la nature $^{25}$. Contre une telle abstraction, qui est un oubli de la vie du sujet et de la vie du monde, Husserl donne à voir que seul un sujet qui s'éprouve dans la singularité originaire de la présence à soi dans le sentir, celle de la chair, peut être disponible à l'altérité des êtres, celle de l'autre homme et celle des choses. On peut même dire que plus la chair est individuée, plus elle est disponible à l'altérité. Tout oubli de la singularité de la vie subjective et de l'altérité du monde conduit à une confusion de soi et du monde où l'altérité des êtres et de soi se trouvent perdues. Certes, on peut s'interroger sur les conditions de l'expérience de cette vie singulière, mais est-il pour autant nécessaire de la faire dériver de l'expérience du non mien ? L'identité personnelle du sujet constituant et l'altérité des choses ne sont-elles pas données de concert? Quoi qu'il en soit de ces questions décisives, il est à nouveau possible de parler de «ma» cogitatio sans renoncer à la réduction phénoménologique puisque tout vécu est inséparable d'un passé propre : «Nous avons appris à comprendre comment la conscience parvient avant toute activité du moi à s'objectiver elle-même, ou comment elle parvient, selon

\footnotetext{
${ }^{22}$ Idées I, § 81, p.275.

${ }^{23}$ Ibid..

${ }^{24}$ Idées I, p.176.

${ }^{25}$ Voir Méditations cartésiennes $§ 44$.
} 
les lois de la genèse originaire, non seulement à posséder en général un passé propre, mais encore à pouvoir prétendre à une connaissance de ce passé propre ${ }^{26} \gg$. De plus, cette mienneté apparait maintenant dans toute sa complexité puisqu'elle se fonde à la fois sur le lien intuitif de la rétention, dans lequel la conscience se retient en quelque sorte elle-même, et sur le pouvoir-être du je pur $^{27}$. La mienneté rétentionnelle précède le je pur comme pouvoir infini de se constituer soi-même et ce je pur donne à voir ce lien intuitif. La constance originaire qui élucide l'expression «soi-même» est donc indissociablement la constance d'une temporalisation passive et la constance d'un pouvoir-être et c'est dans cet entrelacement que se trouve l'individualité originairement constituante.

Propriété et temporalité reconduisent donc l'une à l'autre selon une nécessité eidétique et selon une circularité qui semble bien être l'une des croix de l'analyse phénoménologique. Comment comprendre que la conscience rétentionnelle rende possible une réflexion qui constitue la temporalité immanente ? Le caractère temporel de la conscience fait qu'en quelque sorte elle se précède toujours elle-même. Cette difficulté est loin de disparaître quand l'interrogation se fait plus noétique que noématique. Dans la troisième section des Leçons, Husserl remonte jusqu'au principe de tout objet temporel, à savoir le flux absolu de la conscience qui est constitutif du temps. En effet, si tout objet temporel peut s'écouler plus ou moins rapidement, il présuppose comme sa mesure un flux qui n'est pas un processus. Impression, rétention, protention, comme phénomènes constitutifs du temps, ne sont pas plus ou moins rapides, parce qu'elles ne sont pas dans le temps, mais sont le temps lui-même comme continuité. Husserl précise ce paradoxe : «Dans le flux originaire, il n'y a pas de durée. Car la durée, c'est la forme de quelque chose qui dure ${ }^{28} » . \mathrm{Si}$ un orage est bien une unité de durée, il n'en va pas de même du surgissement continu d'un nouveau maintenant : le temps originaire n'apparaît pas et ne disparaît pas comme un orage, mais est un perpétuel commencement. Faut-il voir là une retombée dans une conception intemporelle de la conscience? Certainement pas, puisqu'il ne s'agit pas de dire que le flux de la conscience absolue n'apparaît pas et donc qu'il n'a pas de temporalité. Husserl veut seulement marquer l'extrême difficulté à dévoiler le mode de donnée propre à la subjectivité absolue. Elle ne se donne pas comme les vécus, elle ne dure pas comme les vécus, et dés lors les noms semblent faire défaut pour décrire son apparaître.

Pour dépasser cette difficulté, Husserl tente de montrer comment le flux absolu constituant se constitue lui-même tout en constituant l'unité des objets temporels. Encore une fois, aucun vécu ne peut être dit mien sans une telle continuité originaire de la présence à soi. De ce point de vue, la temporalité est bien la subjectivité elle-même dans son mode de donnée. Il n'y a pas de conscience intemporelle et la temporalité de la conscience absolue est seulement une autre forme de temporalité qui échappe à la façon habituelle de dire le temps, et qui, pourtant, comme l'a montré Rudolf Bernet, est la forme même de la vie du sujet ${ }^{29}$. Sans nier les difficultés d'une telle présence à soi, qui ne peut pas prendre la forme d'une conscience objectivante, il n'en demeure pas moins que le souci de Husserl est de remonter à une vie originaire qui est irréductible aux vécus du sujet. Dans cette perspective, la non intratemporalité du je pur ne signifie pas non plus une absence de temporalité, mais une

\footnotetext{
${ }^{26}$ De la synthèse passive, p.268.

${ }^{27}$ Il est manifeste que Husserl n'a pas fréquemment pensé ensemble ces deux perspectives, mais on ne peut pas s'appuyer sur la simple distinction des textes qui parlent du temps et ceux qui parlent de l'ego pour y voir une contradiction.

${ }^{28}$ Leçons pour une phénoménologie de la conscience intime du temps, supplément VI, p.151.

${ }^{29}$ Voir La vie du sujet, Paris, PUF, col. Epiméthée, 1994, p.197.
} 
temporalité autre que celle des vécus : «la vie transcendantale et le je transcendantal ne peuvent pas être nés. Seul l'homme dans le monde peut être né. Mais comme je transcendantal, je fus éternellement. Je suis maintenant et à ce maintenant appartient un horizon de passé qui est déroulable à l'infini. Et, justement, cela signifie: je fus éternellement ${ }^{30} »$. Cela signifie qu'au présent vivant appartient un passé sans fin et un futur lointain infiniment ouvert. «Je fus éternellement » exprime donc le pouvoir infini de réactiver le passé dans des synthèses toujours nouvelles. En conséquence, le je transcendantal n'est pas dans le temps, mais il est le site originaire de la temporalisation qui rend possible une individuation du soi. En cela, ne pas être intra-temporel pour le je transcendantal reconduit d'abord à un être constituant et non pas constitué et c'est justement quand on réduit le je au statut d'un étant constitué qu'on lui retire ce qu'il a de plus propre : son pouvoir-être. Ce présent originaire constituant n'est pas lui-même dans le temps, mais il est le flux jaillissant du temps qui est source de toute vie et à partir duquel le sujet s'éprouve dans la singularité de sa temporalisation continue. Husserl ne se situe donc plus ici sous l'horizon de l'ontologie catégoriale, il ne comprend plus ontologiquement le je comme substance puisque ne pas être (intra)-temporel signifie qu'il n'est rien d'étant, mais est le site originaire (Urstand) de toute temporalisation. C'est pourquoi le moi n'a pas une individualité contingente, mais il est «l'individu nécessaire et le seul ${ }^{31}$ ». En effet, le moi vit sa vie originelle, mais sans s'objectiver parce que son être est autre que tout objet.

Cette éternité du je transcendantal manifeste donc le fait que le sujet qui effectue la réduction phénoménologique s'apparaît à lui-même sans référence au monde, c'est-à-dire s'apparaît à lui-même comme vie transcendantale, comme pouvoir infini de temporalisation. Dès lors, la vie transcendantale comme auto-affection du sujet ne peut pas mourir, pas plus qu'elle ne peut naître. La vie en elle-même ne meurt pas et l'éternité du je transcendantal vient en fait signifier l'infinité de sa possibilisation. L'ego transcendantal est en cela antérieur à la distinction de la naissance et de la mort et ce qui les rend possible, de même qu'il est antérieur à la distinction de la veille et du sommeil. Le je transcendantal n'est donc pas singulier et ne peut pas mourir. Seule une chair, c'est-à-dire quelqu'un, peut être une singularité et peut mourir : singularité et mortalité sont indissociables et on ne peut pas plus me retirer ma singularité qu'on ne peut me retirer ma mortalité. Ou alors, au-delà de Husserl, il faudrait penser le fait de me retirer mon pouvoir mourir comme la violence ultimement dépersonnalisante. Quoi qu'il en soit, il y a là un lien eidétique, non élucidé par Husserl, entre singularité et mortalité. A partir de son être devenu, le je transcendantal est bien cet être éternel en devenir qui a toujours la vie devant soi. Ici l'impossibilité de coïncider parfaitement avec soi, de par son existence temporelle, ne remet pas en cause la tâche de rassemblement de soi, puisque cette impossibilité se retourne en possibilité infinie de constitution de soi. Cela assure que le pur apparaître de soi dans sa singularité constituante n'est pas séparable de l'appropriation continue de soi, mais est ce qui confère à cette appropriation son telos. En s'éprouvant immédiatement lui-même comme singulier à partir de sa vie passée, le sujet comprend le travail de constitution précisément comme le sien.

La transcendance dans l'immanence du je montre qu'on ne peut séparer la pure présence à soi de l'ego, qui ne naît ni ne meurt, mais entre en scène et sort de scène, de la présence à soi dans la constitution de la temporalité immanente dans une synthèse passive à partir du présent vivant. Dans le $§ 37$ des Méditations cartésiennes, Husserl justifie l'unité des différentes formes de temporalisation en montrant que le temps est la forme universelle de

\footnotetext{
${ }^{30}$ De la synthèse passive, p.363.

${ }^{31}$ Die Bernauer Manuskripte über das Zeitbewusstsein, Husserliana XXXIII, Kluwer, 2001, p.286. Sur cette question on peut consulter plus généralement les textes $n^{\circ} 14$ et $n^{\circ} 15, p .275-288$.
} 
toute genèse égologique. En voulant penser une causalité transcendantale à partir du concept de motivation, il peut donner à voir que la simple forme de la temporalité relie déjà tous les vécus entre eux puisqu'ils se motivent les uns les autres. Cette première unité entre tous les vécus d'un même flux confirme que la temporalisation est par elle-même individuante et que la simple forme du flux fixe a priori l'horizon des possibles. Si cette simple temporalisation est déjà l'héritage de l'ego dont il est inséparable, elle en rend possible une seconde dans laquelle avec chaque cogito le je acquiert une propriété permanente nouvelle. Le sujet s'apparaît ici dans son propre travail de constitution du monde et cette deuxième forme de la mienneté transcendantale se fonde sur l'histoire sédimentée du sujet et permet d'expliciter le caractère mien du monde. "L'ego qui se constitue pour lui-même dans l'unité d'une histoire $^{32} \gg$ a donc sa façon propre de persister dans le temps dans sa façon de retenir son propre acte. Cependant cette temporalisation de ce que Husserl nomme la monade, c'est-àdire le je constituant qui porte en lui une histoire, rend possible une troisième temporalisation, celle du sujet qui persiste dans le maintien de la validité de ses décisions et dont la figure exemplaire est le philosophe lui-même. Ce qui veille ici n'est pas la seule réflexivité d'un je pur, mais l'identité noétique d'un sujet qui veut vivre dans une constante responsabilité de soi $^{33}$.

Cette fondamentale historicité du sujet, à laquelle Husserl a consacré de longues analyses, et qui s'accomplit dans la temporalisation de la raison, confirme, à travers l'inséparabilité de la responsabilité et de la temporalité, qu'il est possible de penser une identité noétique indépendamment de toute empirie. Le propre de la réduction phénoménologique est de libérer la pensée de toute forme de limitation. Ce thème absolument constant des analyses husserliennes trouve sa confirmation ici puisque l'individuation du soi, comme forme propre de possibilisation infinie, est bien le contraire d'une limitation. En effet, parce qu'elle est une temporalisation, elle est une ouverture, à partir du présent vivant, aux horizons infinis du passé et du futur, elle est une reprise infinie de son histoire. De même, la responsabilité étant une façon d'assumer la temporalité, elle ouvre l'individu à la tâche infinie de porter une idée, qui était là avant lui, et dont il hérite, et qui continuera à s'accomplir après lui. Certes, l'idée éternelle que le philosophe porte n'est jamais seulement la sienne, mais il n'en demeure pas moins que chaque philosophe a une façon absolument propre de la porter. En conséquence, la temporalisation de soi conduit à reconnaître qu'il n'est plus question de s'universaliser en se donnant une tâche infinie contre sa singularité. La singularité est cette fois le mode nécessaire dont le sujet éthique s'apparaît à lui-même : « Nous qui n'avons pas seulement un héritage spirituel, mais qui encore ne sommes de part en part rien d'autre que (de tels) 'devenus' dans l'histoire de l'esprit, nous avons ainsi et ainsi seulement une tâche à accomplir qui nous soit véritablement propre ${ }^{34} »$. La vie éthique n'est pas une vie anonyme et irréelle, celle de tout le monde et de personne, mais elle se donne au contraire comme celle de quelqu'un qui répond «en personne ». La responsabilité à l'égard du sens du monde prend sa signification concrète quand, par un retour à la temporalisation originaire, le monde cesse d'être le monde abstrait des pures et simples choses des sciences de la nature et quand le je qui fait l'expérience du monde cesse d'être un pur sujet théorique lui-même abstrait. Dès lors ma responsabilité est insubstituable et c'est dans ma singularité que je réponds d'un monde qui n'est mien qu'en étant aussi le nôtre.

\footnotetext{
${ }^{32}$ Méditations cartésiennes, trad. franç. M. de Launay, Paris, PUF, 1994, p.123.

${ }^{33}$ Sur l'individuation de soi par un je transcendantal qui n'est pas lui-même individué voir notre ouvrage Personne et sujet selon Husserl, Paris, PUF, col. Epiméthée, 1997.

${ }^{34}$ La crise des sciences européennes et la phénoménologie transcendantale, trad. franç. G. Granel, Paris, Gallimard, p.82.
} 
En conséquence, dire que l'ego constitue le temps que pourtant il présuppose revient à admettre que la voie réflexive prise par Husserl ne consiste pas seulement à s'arracher à un caractère mien du vécu référé à une chose du monde, pour s'en tenir à une singularité issue des décisions libres. Un vécu n'est mien que sur l'horizon d'un passé rétentionnel propre et une tâche n'est mienne que par rapport à une histoire propre sur le fond de laquelle elle m'est confiée. Dans les deux cas il s'agit de reconnaître que la subjectivité porte en elle l'héritage d'un passé qui la constitue et donc qu'elle ne peut pas se constituer simplement par pure identité. De ce fait, ce qui constitue le temps est lui-même temporel et il faudrait alors remettre en cause le projet d'une transparence parfaite à soi qui suppose toujours, finalement, un je intemporel. Le projet d'une connaissance de soi, qui structure l'idéalisme transcendantal, trouverait ici sa limite puisque cette temporalisation originaire de soi, qui singularise absolument, marque que l'épreuve de soi est d'abord l'épreuve d'un excès de ce que l'on est sur ce que l'on pense être. Il y aurait ainsi une obscurité essentielle, non liée à l'ignorance, mais reconduisant à l'impossibilité de surplomber sa propre vie à partir d'un site extra temporel. Il demeure certain que Husserl n'a jamais considéré que le statut de l'être soi pouvait être autre chose qu'une connaissance de soi et une possession de soi, néanmoins, il n'a pas compris le je comme substance parce qu'il a toujours compris le je comme un «je pense quelque chose ». Dès lors, pour Husserl, le monde est bien une détermination essentielle du «je suis » même si ce n'est pas au sens qui est au même moment dégagé par Heidegger ${ }^{35}$. Si le moi est l'orientation vers un objet, cela présuppose la conscience du présent : «Le je ne peut pas s'orienter sans une conscience de ${ }^{36}$. Il faut alors que ce quelque chose appartienne à la teneur interne du je pense et d'une certaine façon en détermine l'être. Dire je c'est toujours s'exprimer en tant qu'être affecté par un flux temporel déjà là.

Une telle perspective s'ouvre à partir de certains textes, quand Husserl s'écarte de sa question directrice, qui est celle du sujet comme fondement de la science, pour s'attacher à décrire en quoi le je transcendantal est aussi l'être qui manifeste une transcendance radicale vis-à-vis de son être constitué. C'est la temporalité même des êtres qui fait que le projet d'une justification absolue de l'être, par un ego qui demeure à l'abri de sa transcendance, ne semble pas pouvoir être tenu jusqu'au bout puisque l'a priori subjectif présuppose finalement toujours l'être temporel pré-donné. Il y a bien en cela une contingence de l'être qui se donne, qui n'est pas réductible au pouvoir de l'ego et qui est lié à sa nature temporelle. La singularité du sujet échappe bien à la distinction ontologique de la substance et de l'accident et possède ainsi un mode de donnée propre que Husserl n'explicite pas vraiment. Quoi qu'il en soit, cette singularité semble difficilement issue du seul pouvoir de l'ego et donc l'ego qui constitue l'être du monde n'en épuise pas le mystère. Or, si la lumière de l'être ne s'épuise pas dans le regard de l'ego, si l'ego dépend de l'être temporel qu'il constitue, cela signifie que même une égologie transcendantale, quand elle veut respecter l'exigence de la donnée, est conduite à reconnaître dans le don même de l'être la limite de son entreprise. La force de la réduction, pour penser le caractère mien du vécu, a justement été de donner à voir une subjectivité absolue, qui est sans référence au monde pour être mieux à l'écoute de l'altérité des choses.

Ne peut-on dire, en langage husserlien, même si Husserl n'a pas développé de telles analyses, que ce caractère mien du vécu ne fait pas l'objet d'une présentation, mais d'une apprésentation? En cela le travail de constitution serait bien l'indication, et non le simple signe, d'une singularité originairement inaccessible. La phénoménologie n'atteint-elle pas ici sa limite en voulant décrire ce qui n'est pas donné ? En réalité, le paradoxe de cette singularité originaire est qu'elle est bien donnée, mais selon un mode radicalement propre. Le passé de

\footnotetext{
${ }^{35}$ Sur cette question, voir notre ouvrage Husserl et l'énigme du monde, Paris, Ed. du Seuil, 2000.

${ }^{36}$ Die Bernauer Manuskripte über das Zeitbewusstsein, p.256.
} 
ma vie intentionnelle que je porte en moi me renvoie à la part inaccessible de moi-même, à cette part qui ne fera jamais l'objet d'une intuition directe. La singularité est bien, cet entrelacs de ce que je me donne, de ce que je me suis donné, dans mon travail de constitution, et de ce qui m'advient dans la pure passivité. Ainsi, en accédant directement à ce que je me donne, j'accède indirectement à ce qu'il m'est donné d'être et qui ne fera jamais l'objet d'un voir apodictique. Certes, je m'apparais bien à moi-même selon une expérience concordante, selon un style propre, mais jamais cela ne supprimera l'énigme de ce que je suis, de ce que je peux être, par rapport à ce que je fus. En conséquence, les paradoxes de la mienneté selon Husserl montrent la nécessité d'une analytique de l'individuation qui montrerait quel est le mode de remplissement intuitif qui est propre à la singularité constituante. Elle se donne comme ne se donnant pas parce qu'elle est donnée à être avant d'être donnée à voir. C'est en cela qu'aucun ressouvenir de ce que je fus, ni aucune anticipation de ce que je serai, ne pourront jamais atteindre la transcendance de ma singularité, qui est une transcendance beaucoup plus radicale et beaucoup plus essentielle que celle de ma vie passée. Tout le paradoxe de la personne est là : ce qui est le plus proprement mien, ma singularité charnelle, ne peut pas par principe être pleinement constituée, même par une intersubjectivité, et en cela elle est à la fois donnée et non donnée. Je suis donné à moi-même comme excédant ce que j'imagine être et pouvoir être et cette altérité à soi n'est pas le symétrique de l'altérité de l'autre homme. En excédant toute saisie et tout projet, elle manifeste ce qu'Henri Maldiney nomme la transpossibilité de l'existence. En cela cette singularité donnée en énigme, énigme qui est ici un mode de l'intentionnalité, est ce qui en moi est le plus essentiellement moimême sans être ce que je me donne : elle est la part inimaginable de moi-même qui m'appelle à être ce que je ne suis pas, c'est-à-dire qui m'appelle à être. Dès lors, ce qui précède l'affirmation de soi, et la rend possible, l'élève à la dignité d'une personne unique et irremplaçable, en la reconduisant à l'identité temporelle d'une tâche infinie, parce que cela lui donne un avenir. La singularité originaire, dans l'obscurité même de sa donation, est l'origine de ma temporalisation parce qu'elle m'appelle à l'impossible de moi-même : elle est en moi ce qui est toujours en départ et ce qui me donne de rencontrer le monde dans le surgissement continu de son altérité. Loin d'être une limitation de mon expérience, elle serait ce qui initie le mouvement de temporalisation et lui donne son telos. Une telle singularité transcendantale serait en cela antérieure à la constitution du tien et du mien et ce qui les rendrait pensables dans la mesure où elle est la vie originaire elle-même qui se révèle comme tâche. En effet, l'ipséité originaire est vie parce qu'elle est reçue et parce qu'elle initie un mouvement en avant qui va à la rencontre de ce qui advient, de ce qui se donne à moi. Ainsi, le paradoxe de la singularité constituante serait d'être cette façon unique d'accueillir, c'est-à-dire de comprendre, ce qui se manifeste et ensuite de le constituer. Ne faut-il pas alors reconnaître que la vie temporelle est tout autre chose qu'une simple particularisation d'une vie universelle anonyme et qu'elle est la façon unique d'accueillir, de dire et de vouloir l'universel ?

Ainsi, être soi revient à assumer la temporalité au lieu de se laisser aller au fil du temps, mais cette possibilité vient elle-même en partie de notre être temporel et dès lors le sujet dans sa liberté éprouve ce qu'il y a d'impossible et d'inutile à vouloir s'approprier pleinement le temps. La constance par laquelle ma vie est précisément la mienne ne peut plus se comprendre comme une simple identité inamissible, qui ne pourrait pas être remise en cause par l'expérience. Certes, pour Husserl, le je transcendantal demeure à l'abri de sa transcendance et jamais Husserl n'envisage une impossibilité de dire je. L'idéalisme transcendantal ne peut pas laisser une place à une suspension de la vie transcendantale par un événement inassumable qui vient bloquer la temporalisation infinie de soi. Néanmoins, pour Husserl, le combat pour le sens du monde suppose une constance propre du sujet, qui n'est 
pas comparable à celle des choses. En effet, on peut se demander si pour faire droit à l'événement de la nouveauté, qui semble manqué par la seule intentionnalité objectivante, il est nécessaire de remettre en cause toute idée de constance. Bien au contraire, ne peut être altéré par ce qui n'est pas lui, ne peut devenir autre dans le cours de l'expérience, que celui qui vit dans l'effort de s'approprier ce qui lui advient. Ne peut être surpris que celui qui cherche à se porter lui-même dans la continuité d'une temporalisation infinie. On peut se demander si une totale disponibilité où l'événement ne viendrait pas briser un effort de constance ne serait pas l'équivalent d'une parfaite fermeture. Certes, en développant la conception d'une temporalisation close sur elle-même, Husserl n'envisage pas que quelque chose d'extérieur puisse bloquer notre pouvoir de possibilisation, mais pour penser une telle discontinuité radicale dans le devenir soi est-il nécessaire de renoncer à toute idée de constance ? Husserl ne fait sans doute pas assez droit à l'altérité de ce qui m'affecte, que ce soit mon propre passé ou le monde, mais il n'en demeure pas moins qu'il a su dégager le caractère essentiellement singulier de la vie transcendantale sans lequel l'expression "soimême » demeure vide. Il lui revient d'avoir montré comment la subjectivité s'éprouve ellemême dans la singularité de sa temporalisation originaire avant toute objectivation active et en quoi la constance de cette épreuve est ce qui rend possible la constance d'une tâche à accomplir comme étant «sa » tâche. Tout en privilégiant la permanence égoïque, Husserl n'oublie pas l'individuation originaire de la vie transcendantale : l'ipséité originaire est la constance de cette retenue du surgissement continu de la nouveauté dans le présent. Ainsi, parce que la vie transcendantale s'éprouve comme déjà mienne dans la temporalisation originaire, elle rend possible l'individuation active d'une vie qui pose des actes libres et qui rencontre le monde en son altérité.

Il ne s'agit pas de méconnaître les considérables difficultés qu'il y a à décrire la singularité de la vie dans l'idéalisme transcendantal husserlien puisqu'en effet, par principe, l'idée que seule l'épreuve de soi enseigne sur sa singularité et que seule l'épreuve des choses enseigne sur leur propre singularité n'est pas compatible avec le transcendantal. Cependant, la transcendance dans l'immanence du je transcendantal, dont on est loin d'avoir encore pris toute la mesure, n'indique-t-elle pas une altérité à soi, un excès de soi sur ce que l'on sait de soi, sans laquelle la singularité de la vie demeure finalement secondaire ? Un tel excès du soi sur la conscience de soi n'est-elle pas ce qui libère d'un enfermement dans la conscience de soi et du même coup ouvre à l'altérité des choses c'est-à-dire à la singularité de leur manifestation? Autrement dit, une phénoménologie de la vie dans sa singularité inamissible ne commande-t-elle pas de dépasser à la fois l'alternative du fait et de l'essence et l'autoaffection dans la mesure où dans les deux cas il y a une absence d'épreuve de l'altérité qui est fondamentalement dépersonnalisante ? L'incarnation est-elle la possibilité a priori de jouir de soi dans une présence totale ou est-elle une venue à soi toujours obscure rendue possible par la vie du monde qui nous advient dans son altérité ? Peut-on décrire la singularité de la vie à partir de l'instantanéité de la réflexivité ou du se sentir ou n'est-elle pas plutôt le temps luimême comme approche de l'altérité des choses dans laquelle nous apprenons à nous connaître?

Cela montre en même temps que la rupture entre Husserl et Heidegger ne consiste pas simplement dans l'opposition entre une psychologie personnaliste et l'analytique existentiale. D'une façon plus précise, Husserl envisage une individuation de soi à partir d'un je transcendantal antérieur à la distinction de la passivité et de l'activité comme à la distinction du tien et du mien. En conséquence, il ne peut pas y avoir pour Husserl de singularisation avant la vie intentionnelle alors que pour Heidegger, comme le marque le $\S 9$ d'Etre et temps, la mienneté est antérieure au pouvoir de dire je et antérieure à ces deux modes de 
temporalisation que sont l'existence propre et l'existence impropre. Il n'y a donc rien de comparable entre une mienneté issue du rapport à soi et une mienneté comme assujettissement à l'être qui fait signe vers l'Ereignis. Husserl décrit en effet, la constance propre du soi, mais sans accéder au souci qui chez Heidegger transforme cette constance en maintien de soi. Il est clair que Husserl ne fonde pas le soi dans le souci, néanmoins la constance élucidée par Husserl ne se distinguera radicalement de la constance liée au souci qu'au moment où Heidegger approfondira sa réflexion sur la mienneté en pensant un homme livré à l'être, en élucidant cette Inständigkeit de l'introduction à la conférence Qu'est-ce que la métaphysique?, Inständigkeit qui est la vérité de l'ipséité chez Heidegger. Cela dit, si le Dasein est lui-même par son rapport essentiel à l'être, on peut se demander s'il est possible de développer pleinement une pensée de la mienneté comme advenir de l'exposition à l'être en dehors d'une philosophie du sentir et de la chair qui elle n'est pas absente chez Husserl. La question demeure alors posée de savoir si c'est la singularisation radicale du Dasein ou la transcendance dans l'immanence de l'ego qui libère le plus sûrement de l'objectivation de soi pour reconduire à la singularité de sa tâche d'être. Au-delà de la distinction bien connue entre la tâche infinie de répondre du sens du monde et la tâche finie de répondre à l'appel de l'être, se pose toujours la question des conditions d'une véritable analytique de l'individuation qui interrogerait pour elle-même cette mienneté passive source de toute ipséité. Finalement, n'estce pas encore un je transcendantal qui peut seul consentir au dessaisissement par l'être pour laisser advenir l'être qui en m'appelant m'individue ? N'est-ce pas dans ce don originaire de soi dans l'incertitude totale de ce que l'on est que l'on peut se recevoir de ce qui nous est donné ? La mienneté n'est-elle pas dans cet ego transcendantal qui loin de demeurer à l'abri de sa transcendance s'expose dans le maintenant et peut alors s'y tenir en s'individuant ? Cependant, ouvrir une telle perspective n'est-ce pas déjà s'éloigner de Husserl comme de Heidegger en déliant la mienneté de toute idée de constance pour la comprendre comme l'excès de ce que je peux être en répondant à autrui qui est la source de mon être ? Ce pouvoir d'être soi n'est-il pas alors radicalement ce que je reçois et non une capacité a priori soit de prendre conscience de soi, soit de faire l'épreuve de l'être ? Avec ces questions il en va du sens d'être de l'individuation : consiste-t-elle à faire sien ce qui advient en s'y possibilisant ou consiste-t-elle à donner ce que je ne suis pas encore ? 